\title{
Study of the Relationship between Serum Asprosin, Endothelial Dysfunction and Insulin Resistance
}

\section{ABSTRACT}

Background: Until now, the relationship between asprosin and soluble E-selectin (sE-selectin) as well as the role of asprosin in diabetes are still unclear. This work aimed to assess the relationship between fasting serum asprosin and markers of endothelial dysfunction and metabolic parameters, namely, sE-selectin, fasting insulin, fasting plasma glucose (FPG), HOmeostatic Model Assesment - Insulin Resistance (HOMA-IR), fasting lipid profile, and glycated hemoglobin (HbA1c) among people with different spectra of glycemia including normal subjects, impaired glucose tolerance (IGR), and subjects with type 2 diabetes mellitus (T2DM).

Methods: Ninety subjects were recruited and divided according to the American Diabetes Association (ADA) diagnostic criteria into three groups; subjects with type 2 diabetes mellitus $(n=30)$, subjects with impaired glucose resistance $(n=30)$, and control subjects $(n=30)$. All groups were subjected to thorough history taking, physical examination, and laboratory investigation, involving fasting asprosin, sE-selectin, and HOMA-IR. Results: The univariate analysis showed a significant positive correlation between asprosin and glycemic parameters, insulin resistance, obesity parameters, and

Address for correspondence:

Mohamed Galal Moawad

Department of Internal Medicine

Alexandria Fever Hospital, University of Alexandria

21 500, Egypt

e-mail: mohamed_galal562003@yahoo.com

Clinical Diabetology 2022, 11; 2: 73-79

DOI: $10.5603 /$ DK.a2022.0006

Received: 22.09.2021

Accepted: 24.10.2021 endothelial dysfunction in the three groups. However, multivariate analysis showed that triglycerides and sE-selectin are the most independent factors affecting asprosin. Univariate analysis for parameters affecting $s E$-selectin showed that asprosin is significantly correlated with $\mathrm{sE}$-selectin, and multivariate analysis revealed that asprosin was the most independent factor affecting sE-selectin.

Conclusions: Our study showed a significant positive correlation between asprosin, glucose dysregulation, insulin resistance, and endothelial dysfunction. (Clin Diabetol 2022, 11; 2: 73-79)

Keywords: asprosin, endothelial dysfunction, souble E-selectin, insulin resistance

\section{Introduction}

Asprosin is a new adipokine [1], secreted by mature white adipose tissue (WAT) [2]. A significant increase in serum asprosin levels in impaired glucose regulation (IGR) and type 2 diabetes mellitus (T2DM) was found. So, the circulating asprosin seems to be able to diagnose early diabetes and also, may be considered as a possible therapeutic goal for prediabetes and T2DM [2].

One of the chief features of insulin resistance (IR) syndrome and/or metabolic syndrome (the portal/visceral hypothesis) is marked central adiposity [3]. Increased adiposity, in particular in visceral depots, causes higher free fatty acid (FFA) flux and insulin action inhibition in insulin-sensitive tissues by Randle's effect [3].

As IR is a direct cause of T2DM, and asprosin occurs with excess adiposity so WAT can lead to glucose 
metabolism modulation and energy homeostasis maintenance, irrespective of enhancement or impairment of insulin action. Also, it seems likely that obesity is dramatically associated with a constellation of T2DM and metabolic syndromes. Therefore, a great role of asprosin in glucose metabolism as well a negative feedback-loop by suppressing circulating asprosin is highly suggested [1, 2].

Endothelial dysfunction (ED) is characterized by high expression of adhesion molecules and abnormal modulation of vascular tone [4]. ED is related to IR states such as diabetes, metabolic syndrome, and obesity [5]. Soluble E-selectin (sE-selectin) is one of the markers of endothelial dysfunction [6]. Plasma sE-selectin concentration may be considered a marker of any change in the endothelial cell whether it was damaged or activated [6].

Up to date, there is a gap in the relationship between the level of asprosin and diagnosis of diabetes and also the relationship between asprosin and soluble E-selectin as a marker of ED.

\section{Methods}

Ninety subjects were recruited and divided according to the American Diabetes Association (ADA) diagnostic criteria [7] into 3 groups; subjects with T2DM ( $n=30)$, subjects with impaired glucose regulation (IGR) $(n=30)$, and control subjects $(n=30)$. The exclusion criteria encompassed the following: subjects with type 1 diabetes mellitus (T1DM), subjects with liver or renal disease, subjects on corticosteroid treatment, and pregnant women.

Ethics Committee of Faculty of Medicine, Alexandria University approved the protocol.

Our Institutional Review Board and Ethical Committee approved the current work which was done according to the Declaration of Helsinki. Informed consent was received from all participants, after counseling them on the nature of the study.

\section{Clinical and biochemical evaluations}

All subjects of the three groups were subjected to detailed history taking, complete physical examination, body mass index (BMI) measurement, and waist circumference (WC) assessment using standard protocols in all subjects.

Laboratory investigation included fasting plasma glucose (FPG), 2 hours post-load plasma glucose during glucose tolerance test (OGTT) (75gm glucose), glycated hemoglobin ( $\mathrm{HbA} 1 \mathrm{C})$, fasting insulin, and homeostatic model assessment (HOMA-IR), fasting lipid profile, fasting asprosin, and sE-selectin.

The collection of peripheral venous blood samples was made in the morning after overnight fasting. By means of centrifugation at 3000 RPM for 15 minutes, serum samples were obtained and then kept at $-20^{\circ} \mathrm{C}$ until used, all within 3 months.

Fasting blood glucose (FBG) and lipid profiles were assayed on Dimension EXL automated chemistry analyzer (Siemens Healthineers, Germany). Glycated hemoglobin was determined by high-performance liquid chromatography. Fasting insulin (FINS) was estimated in serum by chemiluminescent immunoassay technique on ADVIA Centaur (Siemens Healthineers, Germany). The homeostasis model assessment of insulin resistance $(\mathrm{HOMA}-\mathrm{IR})=\mathrm{FPG} \times-\mathrm{FINS} / 22$. Two hours post-load plasma glucose after 75-gram glucose oral ingestion is measured.

Fasting asprosin and sE-selectin levels were detected by commercial enzyme-linked immunosorbent assay (ELISA) kits (MyBioSource, USA) consistent with the manufacturers' instructions. ALL calibration standards were assayed simultaneously as the samples in duplicates and a standard curve of optical density (OD) at $(450 \mathrm{~nm})$ vs. asprosin and sE-selectin concentrations was generated. The concentration in the samples was assessed by comparing the samples' OD to the generated standard curves.

\section{Statistical analysis of the data}

Using IBM SPSS software package version 20.0 (Armonk, NY: IBM Corp), feed and analysis of data to the computer were performed. Description of the qualitative data was done via numbers and percentages. The verification of distribution normality was achieved using the Kolmogorov-Smirnov test. Quantitative data were defined by range (minimum and maximum), mean, standard deviation, median and interquartile range (IQR). The significance of the resulted findings was assessed at the $5 \%$ level [5].

\section{Results}

The main demographic, clinical, and metabolic characteristics of subjects in the 3 groups are illustrated in Table 1. Asprosin (Fig. 1a) was significantly higher in T2DM than in prediabetes and control ( $p<0.001)$. However, E-selectin (Fig. 1b) was significantly higher in T2DM than control but not prediabetes as shown in (Tab. 1). Asprosin was significantly positively correlated with BMI, waist circumference, FBS, postprandial plasma glucose (PPPG), HbA1c, HOMA-IR (Fig. 2a), triglycerides (TG), and E-selectin. (Tab. 2) Also, E-selectine was significantly positively correlated with FBG, PPPG, HbA1c, HOMA-IR (Fig. 2b), and asprosin, but negatively correlated with HDL-c (Tab. 3).

Multivariate analysis shows that TG and E-selectin are the two independent factors affecting asprosin (Tab. 4). 
Table 1. Comparison between the Different Groups Aaccording to Demographic, Clinical and Biochemical Parameters

\begin{tabular}{|c|c|c|c|c|c|}
\hline & $\begin{array}{l}\text { Group } 1 \\
(n=30)\end{array}$ & $\begin{array}{l}\text { Group } 2 \\
(n=30)\end{array}$ & $\begin{array}{l}\text { Group } 3 \\
(n=30)\end{array}$ & Test of sig. & $\mathbf{P}$ \\
\hline \multicolumn{6}{|c|}{ Demographic and clinical data } \\
\hline Age [years] & $49.07 \pm 7.01$ & $50.67 \pm 11.42$ & $46.87 \pm 8.07$ & $\mathrm{~F}=1.338$ & 0.268 \\
\hline BMI $\left[\mathrm{kg} / \mathrm{m}^{2}\right]$ & $32(29.8-35.3)$ & $30.7(28.4-34.2)$ & $30.45(29.1-32.4)$ & $H=0.806$ & 0.668 \\
\hline$W C[\mathrm{~cm}]$ & $107(100-117)$ & $105(102-110)$ & & & \\
\hline $104(100-111)$ & $\mathrm{H}=1.284$ & 0.526 & & & \\
\hline \multicolumn{6}{|l|}{ Glycemic parameters } \\
\hline FBG $[\mathrm{mg} / \mathrm{dL}]$ & $233(149-311)$ & $113.5^{\mathrm{a}}(110-118)$ & $97.5^{\mathrm{ab}}(93-99)$ & $H=75.435^{*}$ & $<0.001 *$ \\
\hline PPG [mg/dL] & $358.70 \pm 102.42$ & $149.60^{\mathrm{a}} \pm 25.06$ & $112.53^{\mathrm{a}} \pm 16.83$ & $F=139.05^{*}$ & $<0.001 *$ \\
\hline $\mathrm{HbA} 1 \mathrm{c}[\%]$ & $10.17 \pm 2.44$ & $5.94^{\mathrm{a}} \pm 0.24$ & $5.40^{\mathrm{a}} \pm 0.37$ & $\mathrm{~F}=100.41^{*}$ & $<0.001 *$ \\
\hline Fasting insulin $[\mu \mathrm{U} / \mathrm{L}]$ & $12.2(9.2-18.7)$ & $9.19(8.5-13.73)$ & 10.65(9.3-13.3) & $H=5.259$ & 0.072 \\
\hline HOMA-IR & $8.56 \pm 4.56$ & $3.09^{\mathrm{a}} \pm 1.29$ & $2.10^{\mathrm{a}} \pm 0.73$ & $F=47.468^{*}$ & $<0.001 *$ \\
\hline \multicolumn{6}{|l|}{ Lipid profile [mg/dL] } \\
\hline Total cholesterol & $232.67 \pm 54.25$ & $228.53 \pm 45.58$ & $205.1^{\mathrm{a}} \pm 47.56$ & $F=2.738$ & 0.070 \\
\hline LDL & $152.5(125-199)$ & $148.5(120-172)$ & $121.5^{\mathrm{a}}(97-167)$ & $\mathrm{H}=7.333^{*}$ & $0.026^{*}$ \\
\hline HDL & $38.5(34-56)$ & $47.5(40-58)$ & $49(42-56)$ & $H=3.507$ & 0.173 \\
\hline TG & $146(96-215)$ & $149.5(92-247)$ & $99.5^{\mathrm{ab}}(81-153)$ & $H=6.480^{*}$ & $0.039^{*}$ \\
\hline Asprosin [ng/mL] & $15.32 \pm 4.96$ & $12.42^{\mathrm{a}} \pm 4.40$ & $10.9^{a} \pm 2.74$ & $\mathrm{~F}=8.831^{*}$ & $<0.001 *$ \\
\hline sE-selectin (ng/ml) & $314.54 \pm 53.30$ & $286.14 \pm 86.34$ & $267.97 a \pm 65.48$ & $\mathrm{~F}=3.400^{*}$ & $0.038^{*}$ \\
\hline
\end{tabular}

Normally quantitative data was expressed as mean \pm standard deviation. and compared using F ANOVA test. Abnormally quantitative data was expressed as median (interquartile range) and compared using Kruskal Wallis test. aStatistically significant with group 1; bStatistically significant with group 2; Group 1: subjects with type 2 diabetes mellitus; Group 2: subjects with impaired glucose regulation; Group 3: normal subjects

$\mathrm{BMI}$ - body mass index; FBG — fasting plasma glucose; HbA1c — glycated hemoglobin; HDL — high-density lipoprotein; HOMA-IR — Homeostatic Model Assessment of Insulin Resistance; LDL — low-density lipoprotein; PPG — postprandial glucose; sE-selectin — soluble E-selectin; TG — triglycerides; WC — waist circumference

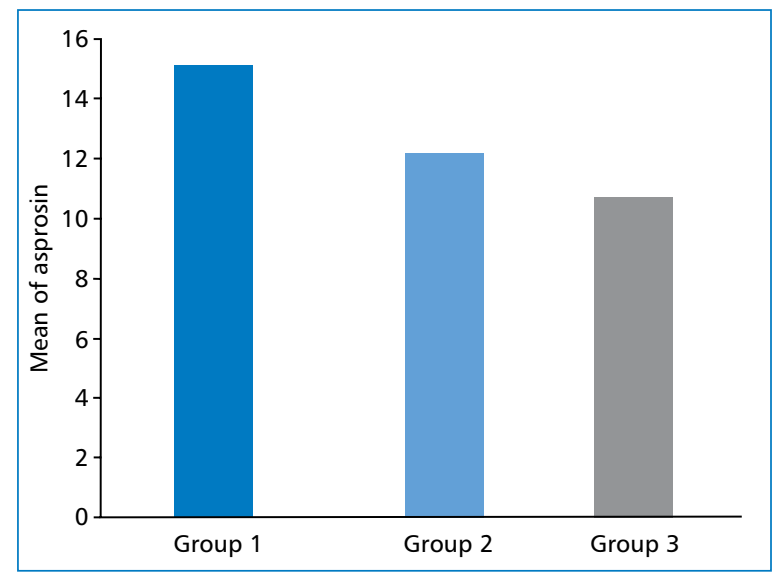

Figure 1a. Comparison between the Different Groups According to Asprosin

While asprosin was the single independent factor affecting E-selectin (Tab. 5).

\section{Discussion}

Romere et al. [1] reported that WAT is the place of asprosin secretion. Under physiological conditions, circulating asprosin acts as a sensor of glucose

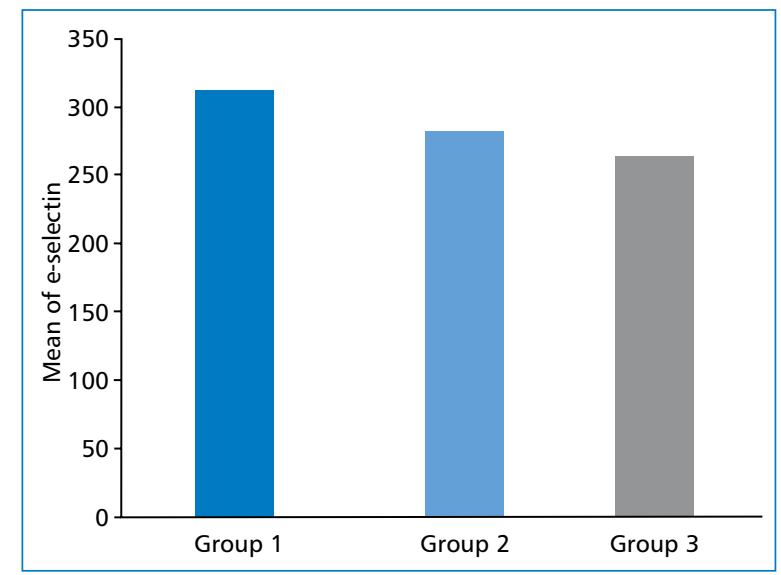

Figure 1b. Comparison between the Different Groups According to E-selectin

that regulates the plasma glucose level via targeting the liver. Besides, they found circulating asprosin in a small number of patients with IR. Concerning asprosin physiological function and its significant association with IR (HOMA-IR), T2DM, and endothelial dysfunction (E-selectin), we reported these associations in the three collected groups. 


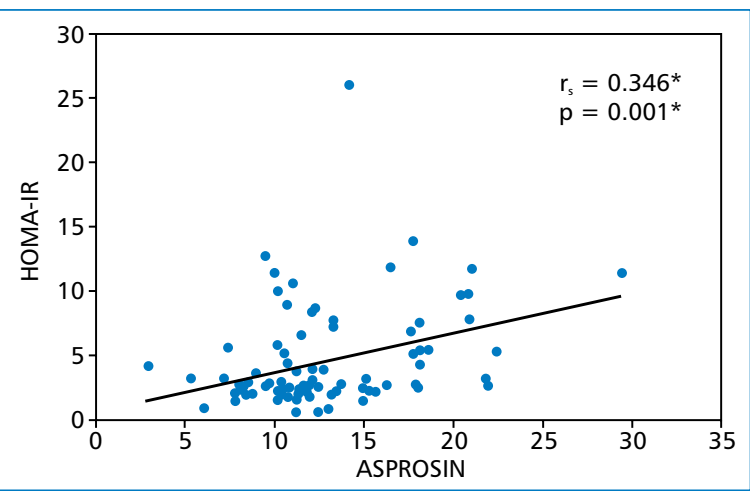

Figure 2a. Comparison between the Different Groups According to Asprosin

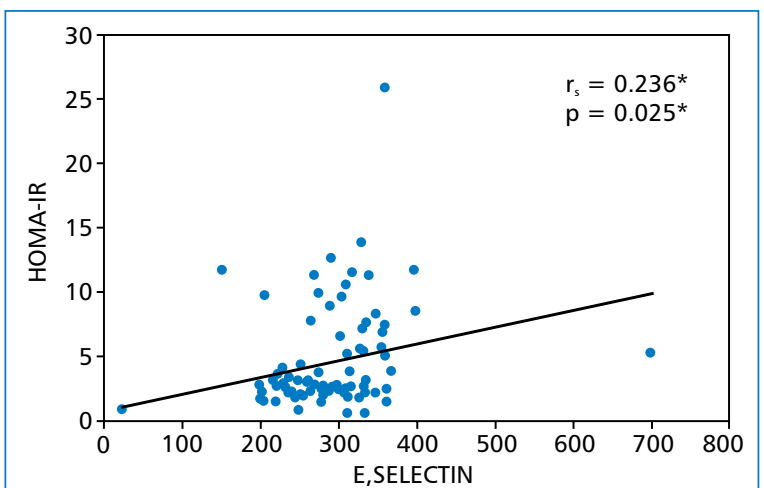

Figure 2b. Comparison between the Different Groups According to E-selectin

Table 2. Correlation between Asprosin and Different Parameters $(n=90)$

\begin{tabular}{|c|c|c|c|c|c|c|c|c|}
\hline & \multicolumn{8}{|c|}{ Asprosin } \\
\hline & \multicolumn{2}{|c|}{ Total $(n=90)$} & \multicolumn{2}{|c|}{ Group $1(n=30)$} & \multicolumn{2}{|c|}{ Group $2(n=30)$} & \multicolumn{2}{|c|}{ Group $3(n=30)$} \\
\hline & $r$ & $\mathbf{p}$ & $r$ & $p$ & $r$ & $p$ & $r$ & $\mathbf{p}$ \\
\hline Age [years] & -0.156 & 0.141 & -0.336 & 0.070 & -0.281 & 0.132 & -0.056 & 0.770 \\
\hline BMI $\left[\mathrm{kg} / \mathrm{m}^{2}\right]$ & $0.221 *$ & $0.036^{*}$ & $0.956^{*}$ & $<0.001 *$ & -0.352 & 0.057 & -0.144 & 0.447 \\
\hline WC $[\mathrm{cm}]$ & $0.307^{*}$ & $0.003^{*}$ & $0.711^{*}$ & $<0.001^{*}$ & -0.123 & 0.517 & -0.140 & 0.461 \\
\hline FBG [mg/dL] & $0.385^{*}$ & $<0.001^{*}$ & 0.153 & 0.420 & 0.234 & 0.213 & -0.086 & 0.652 \\
\hline PPG [mg/dL] & $0.327^{*}$ & $0.002^{*}$ & 0.091 & 0.633 & 0.040 & 0.832 & -0.059 & 0.759 \\
\hline HbA1c [\%] & $0.317^{*}$ & $0.002^{*}$ & 0.123 & 0.517 & -0.256 & 0.172 & -0.020 & 0.918 \\
\hline Fasting insulin $[\mu \mathrm{U} / \mathrm{L}]$ & -0.012 & 0.907 & -0.014 & 0.943 & 0.088 & 0.642 & -0.281 & 0.132 \\
\hline HOMA-IR & $0.288^{*}$ & $0.006^{*}$ & 0.043 & 0.823 & 0.134 & 0.481 & -0.241 & 0.200 \\
\hline Total cholesterol [mg/dL] & -0.116 & 0.277 & -0.182 & 0.336 & -0.084 & 0.659 & -0.340 & 0.066 \\
\hline LDL & -0.034 & 0.754 & -0.098 & 0.606 & -0.064 & 0.736 & -0.301 & 0.106 \\
\hline HDL & -0.144 & 0.174 & 0.074 & 0.697 & -0.012 & 0.948 & -0.211 & 0.264 \\
\hline TG $[\mathrm{mg} / \mathrm{dL}]$ & $0.530^{*}$ & $<0.001^{*}$ & $0.863^{*}$ & $<0.001^{*}$ & -0.012 & 0.951 & -0.229 & 0.224 \\
\hline E-selectin[ng/dL] & $0.359^{*}$ & $0.001 *$ & -0.186 & 0.325 & $0.601^{*}$ & $<0.001^{*}$ & $0.382^{*}$ & $0.037^{*}$ \\
\hline
\end{tabular}

*Statistically significant at $\mathrm{p} \leq 0.05$; Group 1: subjects with type 2 diabetes mellitus; Group 2: subjects with impaired glucose regulation; Group 3: normal subjects

BMI — body mass index; FBG — fasting plasma glucose; HbA1c - glycated hemoglobin; HDL — high-density lipoprotein; HOMA-IR — Homeostatic Model Assessment of Insulin Resistance; LDL — low-density lipoprotein; PPG — postprandial glucose; $r$ - Pearson coefficient; sE-selectin — soluble E-selectin; TG — triglycerides; WC — waist circumference

In agreement with Zhang et al. [6], Wang et al. [2] and Li et al. studies [8], our study showed that asprosin is significantly higher in T2DM than IGR and normal glucose tolerance (NGR) with a linear increase from NGR to IGR to T2DM. On the contrary, Wang et al. study [2] showed that asprosin was higher in the IGR group. In addition, the study demonstrated that plasma asprosin levels were significantly correlated with different parameters including IR, glucose metabolism, lipid profiles, and obesity.

A preceding study reported that the increased plasma asprosin level was detected in pathological conditions in human beings and also in animal models with genetic-and diet-induced IR [1]. Similarly, our study demonstrated a positive correlation between plasma asprosin levels and IR (HOMA-IR) and a negative correlation with fasting insulin, revealing the dysregulation of asprosin-related glucose could be due to its role in IR and asprosin mediated TLR4/JNK-dependent pathway B cell malfunction [9]. Furthermore, Zhang et al. [6] and Romere et al. [1] studies have found that asprosin directly affects hepatocyte glucose production with no possible compensatory effect by insulin.

In the current study, asprosin, in the whole study population, was significantly positively correlated with FBG, postprandial blood glucose, and $\mathrm{HbA} 1 \mathrm{c}$. That was 
Table 3. Correlation between E-selectin and Different Parameters $(n=90)$

\begin{tabular}{|c|c|c|c|c|c|c|c|c|}
\hline & \multicolumn{8}{|c|}{ sE-selectin } \\
\hline & \multicolumn{2}{|c|}{ Total $(n=90)$} & \multicolumn{2}{|c|}{ Group $1(n=30)$} & \multicolumn{2}{|c|}{ Group $2(n=30)$} & \multicolumn{2}{|c|}{ Group $3(n=30)$} \\
\hline & $\mathbf{r}$ & $\mathbf{p}$ & $\mathbf{r}$ & $\mathbf{p}$ & $\mathbf{r}$ & $p$ & $r$ & $\mathbf{p}$ \\
\hline Age [years] & 0.069 & 0.520 & 0.155 & 0.415 & -0.227 & 0.227 & 0.246 & 0.190 \\
\hline BMI $\left[\mathrm{kg} / \mathrm{m}^{2}\right]$ & -0.198 & 0.062 & -0.359 & 0.052 & -0.303 & 0.104 & -0.009 & 0.964 \\
\hline WC $[\mathrm{cm}]$ & -0.100 & 0.347 & -0.317 & 0.088 & -0.159 & 0.400 & -0.161 & 0.395 \\
\hline $\mathrm{FBG}[\mathrm{mg} / \mathrm{dL}]$ & $0.339 *$ & $0.001^{*}$ & -0.011 & 0.954 & $0.387^{*}$ & $0.035^{*}$ & 0.087 & 0.648 \\
\hline PPG $[\mathrm{mg} / \mathrm{dL}]$ & $0.324^{*}$ & $0.002 *$ & 0.011 & 0.953 & 0.036 & 0.852 & 0.023 & 0.903 \\
\hline $\mathrm{HbA1c}[\%]$ & $0.353^{*}$ & $0.001 *$ & 0.120 & 0.528 & -0.033 & 0.861 & 0.183 & 0.332 \\
\hline Fasting insulin $[\mu \mathrm{U} / \mathrm{L}]$ & 0.107 & 0.317 & 0.066 & 0.728 & 0.017 & 0.928 & 0.153 & 0.420 \\
\hline HOMA-IR & $0.285^{*}$ & $0.006^{*}$ & -0.030 & 0.874 & 0.039 & 0.839 & -0.065 & 0.732 \\
\hline Total cholesterol [mg/dL] & 0.036 & 0.739 & -0.351 & 0.057 & 0.125 & 0.512 & 0.141 & 0.459 \\
\hline LDL & 0.061 & 0.570 & $-0.407^{*}$ & $0.026^{*}$ & 0.127 & 0.502 & 0.163 & 0.390 \\
\hline HDL & $-0.233^{*}$ & $0.027^{*}$ & -0.136 & 0.473 & -0.097 & 0.609 & $-0.368 *$ & $0.045^{*}$ \\
\hline $\mathrm{TG}[\mathrm{mg} / \mathrm{dL}]$ & 0.001 & 0.993 & -0.358 & 0.052 & 0.088 & 0.642 & -0.034 & 0.859 \\
\hline Asprosin [ng/dL] & $0.359 *$ & $0.001 *$ & -0.186 & 0.325 & $0.601 *$ & $<0.001 *$ & $0.382 *$ & $0.037^{*}$ \\
\hline
\end{tabular}

*Statistically significant at $\mathrm{p} \leq 0.05$; Group 1: subjects with type 2 diabetes mellitus; Group 2: subjects with impaired glucose regulation; Group 3: normal subjects

$\mathrm{BMI}$ - body mass index; FBG — fasting plasma glucose; HbA1c — glycated hemoglobin; HDL — high-density lipoprotein; HOMA-IR — Homeostatic Model Assessment of Insulin Resistance; LDL — low-density lipoprotein; PPG — postprandial glucose; $r$ — Pearson coefficient; sE-selectin — soluble E-selectin; TG — triglycerides; WC — waist circumference

Table 4. Univariate and Multivariate Analysis for the Parameters Affecting Asprosin for Total Sample $(\mathrm{n}=90)$

\begin{tabular}{|c|c|c|c|c|}
\hline \multirow[t]{2}{*}{ Asprosin } & \multicolumn{2}{|c|}{ Univariate } & \multicolumn{2}{|c|}{ Multivariate } \\
\hline & $\mathbf{P}$ & B $(95 \% \mathrm{Cl})$ & $\mathbf{P}$ & B $(95 \% \mathrm{CI})$ \\
\hline Females & 0.534 & $-0.598(-2.497-1.302)$ & & \\
\hline Age [years] & 0.191 & $-0.069(-0.173-0.035)$ & & \\
\hline BMI $\left[\mathrm{kg} / \mathrm{m}^{2}\right]$ & $0.036 *$ & $0.168 *(0.011-0.326)$ & 0.290 & $0.114(-0.099-0.328)$ \\
\hline WC $[\mathrm{cm}]$ & $0.003^{*}$ & $0.122 *(0.042-0.203)$ & 0.819 & $-0.014(-0.136-0.108)$ \\
\hline Family history of DMT2 & 0.224 & $1.164(-0.724-3.052)$ & & \\
\hline $\mathrm{FBG}[\mathrm{mg} / \mathrm{dl}]$ & $<0.001 *$ & $0.019 *(0.010-0.029)$ & 0.749 & $0.004(-0.021-0.028)$ \\
\hline PPG [mg/dl] & $<0.001^{*}$ & $0.013(0.006-0.020)$ & 0.906 & $0.001(-0.016-0.018)$ \\
\hline $\mathrm{HbA} 1 \mathrm{c}$ & $0.001^{*}$ & $0.622(0.276-0.968)$ & 0.745 & $0.124(-0.631-0.879)$ \\
\hline Fasting insulin $[\boldsymbol{\mu U} / \mathrm{L}]$ & 0.859 & $0.013(-0.131-0.157)$ & & \\
\hline HOMA-IR & $0.001 *$ & $0.393(0.167-0.619)$ & 0.603 & $0.071(-0.199-0.341)$ \\
\hline Total cholesterol [mg/dl] & 0.619 & $-0.005(-0.024-0.014)$ & & \\
\hline LDL & 0.341 & $0.005(-0.005-0.015)$ & & \\
\hline HDL & 0.875 & $-0.003(-0.046-0.039)$ & & \\
\hline $\mathrm{TG}[\mathrm{mg} / \mathrm{dL}]$ & $<0.001 *$ & $0.019 *(0.013-0.026)$ & $<0.001 *$ & $0.016 *(0.009-0.024)$ \\
\hline E-selectin [ng/dL] & $0.002 *$ & $0.021(0.008-0.033)$ & $0.001 *$ & $0.019 *(0.008-0.030)$ \\
\hline
\end{tabular}

\#All variables with $p<0.05$ was included in the multivariate; * Statistically significant at $p \leq 0.05$

B - standardized coefficients; BMI — body mass index; $\mathrm{Cl}$ — confidence interval; FBG — fasting plasma glucose; HbA1c - glycated hemoglobin; HDL — high-density lipoprotein; HOMA-IR - Homeostatic Model Assessment of Insulin Resistance; LDL — low-density lipoprotein; PPG — postprandial glucose;

$\mathrm{r}$ - Pearson coefficient; sE-selectin — soluble E-selectin; TD2DM - type 2 diabetes mellitus; TG — triglycerides; WC - waist circumference

similar to the findings demonstrated by Zhang et al. [6], Wang et al. [2], and Li et al. [8]. So, asprosin may be an early biomarker of T2DM.

In the diabetic group of our study, asprosin was significantly positively correlated with all glycemic parameters. The same applies to the IGR group except for HbA1c. Zhang et al. [6] had only reported the above-mentioned relationships in T2DM patients, but not in participants with NGT. However, in another recent study by Zhang et al. [10] also, when they 
Table 5. Univariate and Multivariate Analysis for the Parameters Affecting E-selectin

\begin{tabular}{|c|c|c|c|c|}
\hline \multirow[t]{2}{*}{ E-selectin (ng/dL) } & \multicolumn{2}{|c|}{ Univariate } & \multicolumn{2}{|c|}{ \#Multivariate } \\
\hline & $\mathbf{P}$ & B $(95 \% \mathrm{Cl})$ & $\mathbf{P}$ & B $(95 \% \mathrm{Cl})$ \\
\hline Females & 0.105 & $-24.65(-54.54-5.243)$ & & \\
\hline Age [years] & 0.728 & $0.293(-1.378-1.964)$ & & \\
\hline BMI $\left[\mathrm{kg} / \mathrm{m}^{2}\right]$ & 0.062 & $-2.403(-4.92-0.119)$ & & \\
\hline WC $[\mathrm{cm}]$ & 0.347 & $-0.637(-1.975-0.701)$ & & \\
\hline Family history of T2DM & 0.161 & $21.361(-8.646-51.368)$ & & \\
\hline $\mathrm{FBG}[\mathrm{mg} / \mathrm{dL}]$ & 0.057 & $0.162(-0.005-0.329)$ & & \\
\hline PPG [mg/dL] & $0.029 *$ & $0.132(0.014-0.250)$ & 0.904 & $0.016(-0.251-0.284)$ \\
\hline $\mathrm{HbA} 1 \mathrm{c} \%$ & $0.030 *$ & $6.376(0.633-12.118)$ & 0.855 & $1.201(-11.829-14.23)$ \\
\hline Fasting insulin $[\boldsymbol{\mu} \mathrm{U} / \mathrm{L}]$ & 0.246 & $1.338(-0.940-3.617)$ & & \\
\hline HOMA-IR & $0.025^{*}$ & $4.277(0.551-8.004)$ & 0.512 & $1.703(-3.44-6.845)$ \\
\hline Total cholesterol [mg/dL] & 0.803 & $-0.038(-0.340-0.264)$ & & \\
\hline LDL & 0.678 & $-0.034(-0.195-0.127)$ & & \\
\hline HDL & 0.356 & $-0.316(-0.993-0.361)$ & & \\
\hline $\mathrm{TG}[\mathrm{mg} / \mathrm{dL}]$ & 0.993 & $0.001(-0.123-0.124)$ & & \\
\hline Asprosin [ng/dL] & $0.002 *$ & $5.236(2.046-8.425)$ & $0.016 *$ & $4.306(0.817-7.795)$ \\
\hline
\end{tabular}

\#All variables with $p<0.05$ was included in the multivariate; *Statistically significant at $p \leq 0.05$

B - standardized coefficients; BMI — body mass index; $\mathrm{Cl}$ - confidence interval; FBG — fasting plasma glucose; HbA1c — glycated hemoglobin; HDL — high-density lipoprotein; HOMA-IR - Homeostatic Model Assessment of Insulin Resistance; LDL — low-density lipoprotein; PPG — postprandial glucose; $r$ - Pearson coefficient; sE-selectin — soluble E-selectin; TD2DM - type 2 diabetes mellitus; TG — triglycerides; WC — waist circumference

analyzed participants with normal glucose tolerance (NGT) or T2DM distinctly, no association between fasting asprosin level and glycemic parameters was recorded in both groups apart from $\mathrm{HbA} 1 \mathrm{c}$ in patients with NGT [10].

The contradictory findings between our study and the recent Zhang et al. [10] study may be due to the inclusion of long-standing T2DM patients in their study. The dysregulation of asprosin secretion by WAT in newly diagnosed T2DM patients causes a pathologically increased asprosin concentration. However, with the longstanding of T2DM, this dysregulation might be worse and may have an effect asprosin response to glucose fluctuation, which results in the impaired relationship between fasting circulating asprosin level and glucose metabolism parameters in this cohort of patients.

In the current study, asprosin was significantly correlated with TG in the three groups including diabetic patients but was not correlated with total cholesterol in the three groups as in Wang et al. [2]. On the contrary, Zhang et al. [10] showed that asprosin is positive significantly correlated with all lipid parameters.

In our study, TG and E-selectin were the two independent factors associated with asprosin levels in the three groups, however, in Zhang et al. [6] cohort study, TG, and FBG were the independent factors associated with asprosin concentrations in T2DM.

In our study and two Chinese studies $[2,6]$ asprosin was significantly correlated with obesity parameters
(BMI, WC) in the three groups including the diabetic group that points to the possible relationship between asprosin, IR, and adiposity. In comparison, multiple stepwise regression analysis did not report any independent association between these adiposity indices and adipokines. In the same line, a recent study found that in T2DM, the serum adiponectin alternation and chemerin concentrations did not relate to obesity [11]. This could be explained by the inequality between the adipokines amount within the tissue and the quantity released into the circulation [12]. Besides, despite asprosin is principally secreted by WAT, it also secreted by other tissues [1]. Therefore, the association between different parameters of obesity and concentrations of asprosin may be underestimated.

In the current study, E-selectin, a marker of endothelial function, had a significant positive correlation with all glycemic parameters (FBG, PPPG, HbA1c, HOMA-IR), insulin resistance, and asprosin. Asprosin was the single independent factor affecting E-selectin (Tab. 5). The positive relationship between asprosin, $I R$, and endothelial dysfunction explains the clustering of metabolic and cardiovascular diseases in IR states as described by Poirier P.et al. study [13]. Hence, E-selectin can be thought of as a predictor of cardiovascular diseases such as peripheral vascular disease, coronary artery disease, and stroke.

Our study has some limitations; first, it is a crosssectional study that didn't confirm a causal relationship 
between the occurrence of diabetes (or prediabetes), asprosin, and endothelial dysfunction. Secondly, it included one ethnic group.

\section{Conclusions}

Our study showed a positive correlation between asprosin, glucose dysregulation, insulin resistance, endothelial dysfunction, and dyslipidemia.

We recommend further studies assessing asprosin as a therapeutic target in T2DM.

\section{Acknowledgments}

The authors of this study possess no acknowledgments to make with regards to this study and this manuscript's content.

\section{Funding}

Internal Medicine Department and Clinical Pathology Department, Faculty of Medicine, Alexandria University

\section{Conflict of interest}

None declared.

\section{REFERENCES}

1. Romere C, Duerrschmid C, Bournat J, et al. Asprosin, a fasting-induced glucogenic protein hormone. Cell. 2016; 165(3): 566-579, doi: 10.1016/j.cell.2016.02.063, indexed in Pubmed: 27087445.

2. Wang $\mathrm{Y}, \mathrm{Qu} \mathrm{H}$, Xiong $\mathrm{X}$, et al. Plasma asprosin concentrations are increased in individuals with glucose dysregulation and correlated with insulin resistance and first-phase insulin secretion. Mediators Inflamm. 2018; 2018: 9471583, doi: 10.1155/2018/9471583, indexed in Pubmed: 29743813.

3. Ronti T, Lupattelli G, Mannarino $E$. The endocrine function of adipose tissue: an update. Clin Endocrinol (Oxf). 2006; 64(4): 355-365, doi: 10.1111/j.1365-2265.2006.02474.x, indexed in Pubmed: 16584505.

4. Kim Ja, Montagnani M, Koh KK, et al. Reciprocal relationships between insulin resistance and endothelial dysfunction: molecular and pathophysiological mechanisms. Circulation. 2006; 113(15): 1888-1904, doi: 10.1161/CIRCULATIONAHA.105.563213, indexed in Pubmed: 16618833.

5. Daiber A, Steven S, Weber A, et al. Targeting vascular (endothelial) dysfunction. Br J Pharmacol. 2017; 174(12): 1591-1619, doi: 10.1111/bph.13517, indexed in Pubmed: 27187006.

6. Zhang L, Chen C, Zhou N, et al. Circulating asprosin concentrations are increased in type 2 diabetes mellitus and independently associated with fasting glucose and triglyceride. Clin Chim Acta. 2019; 489: 183-188, doi: 10.1016/j.cca.2017.10.034, indexed in Pubmed: 29104036.

7. Davies MJ, D'Alessio DA, Fradkin J, et al. Management of hyperglycemia in type 2 diabetes, 2018. A consensus report by the American Diabetes Association (ADA) and the European Association for the Study of Diabetes (EASD). Diabetes Care. 2018; 41(12): 2669-2701, doi: 10.2337/dci18-0033, indexed in Pubmed: 30291106.

8. Li X, Liao M, Shen R, et al. Plasma asprosin levels are associated with glucose metabolism, lipid, and sex hormone profiles in females with metabolic-related diseases. Mediators Inflamm. 2018; 2018: 7375294, doi: 10.1155/2018/7375294, indexed in Pubmed: 30524197.

9. Lee $\mathrm{T}$, Yun $\mathrm{S}$, Jeong JiH, et al. Asprosin impairs insulin secretion in response to glucose and viability through TLR4/JNK-mediated inflammation. Mol Cell Endocrinol. 2019; 486: 96-104, doi: 10.1016/j.mce.2019.03.001, indexed in Pubmed: 30853600.

10. Zhang $X$, Jiang $H, M a X$, et al. Increased serum level and impaired response to glucose fluctuation of asprosin is associated with type 2 diabetes mellitus. J Diabetes Investig. 2020; 11(2): 349-355, doi: 10.1111/jdi.13148, indexed in Pubmed: 31529619.

11. Andersson DP, Laurencikiene J, Acosta JR, et al. Circulating and adipose levels of adipokines associated with insulin sensitivity in nonobese subjects with type 2 diabetes. J Clin Endocrinol Metab. 2016; 101(10): 3765-3771, doi: 10.1210/jc.2016-1883, indexed in Pubmed: 27501281.

12. Kovács $D$, Lovászi $M$, Póliska $S$, et al. Sebocytes differentially express and secrete adipokines. Exp Dermatol. 2016; 25(3): 194-199, doi: 10.1111/exd.12879, indexed in Pubmed: 26476096.

13. Poirier P, Giles TD, Bray GA et al. American Heart Association, Obesity Committee of the Council on Nutrition, Physical Activity, and Metabolism. Obesity and cardiovascular disease: pathophysiology, evaluation, and effect of weight loss: an update of the 1997 American Heart Association Scientific Statement on Obesity and Heart Disease from the Obesity Committee of the Council on Nutrition, Physical Activity, and Metabolism. Circulation. 2006; 113(6): 898-918, doi: 10.1161/CIRCULATIONAHA.106.171016, indexed in Pubmed: 16380542. 